\title{
A Method Of Separation Assurance FOR INSTRUMENT FLIGHT PROCEDURES AT NON-RADAR AIRPORTS
}

\author{
Sheila R. Conway \\ Langley Research Center \\ National Aeronautics and Space Administration \\ Hampton, VA \\ Maria Consiglio \\ ICASE \\ Universities Space Research Association \\ Hampton, VA
}

\section{Abstract}

A method to provide automated air traffic separation assurance services during approach to or departure from a non-radar, non-towered airport environment is described. The method is constrained by provision of these services without radical changes or ambitious investments in current ground-based technologies. The proposed procedures are designed to grant access to a large number of airfields that currently have no or very limited access under Instrument Flight Rules (IFR), thus increasing mobility with minimal infrastructure investment. This paper primarily addresses a low-cost option for airport and instrument approach infrastructure, but is designed to be an architecture from which a more efficient, albeit more complex, system may be developed.

A functional description of the capabilities in the current NAS infrastructure is provided. Automated terminal operations and procedures are introduced. Rules of engagement and the operations are defined. Results of preliminary simulation testing are presented. Finally, application of the method to more terminal-like operations, and major research areas, including necessary piloted studies, are discussed.

\section{Introduction}

In the past few years the limitations of the existing air transport system have become obvious. Frequent flight delays and cancellations, with all the attendant stress and disruption, are now a familiar part of air travel in the United States. Many of these difficulties result from the dominant hub-and-spoke model that requires concentration of a large percentage of air traffic at a few airports. Despite the implementation of improved air traffic management tools and construction of new runways, it has become clear that significant increases in overall air traffic will soon make demands on these airports that simply cannot be satisfied by increasing capacity. Moreover, the current focus on capacity problems at hub airports has tended to obscure another fundamental deficiency in the hub-and-spoke system: the need to change planes. No one traveling from Milwaukee to Shreveport really wants to drag their carry-on luggage through the terminal at O'Hare; they do so because there is no direct flight available at reasonable cost. Finally, though most people live in large metropolitan areas serviced by large international airports, there are also many people who would find travel to a small or metro-satellite airport more appealing. Unfortunately at these smaller airports, viable, cost competitive air transportation is not available.

Because increasing capacity alone does not appear to provide a long-term solution to the problem of delays or satisfy the demand for more direct flights, another line of research has emerged which is aimed at increasing the "mobility" of the system, meaning its ability to accommodate larger numbers of on-demand, point-to-point IFR operations between smaller airports. This approach complements efforts to increase capacity by promoting more evenly distributed air traffic and reducing congestion at large hub airports. A number of technologies show promise in increasing mobility: The use of Automatic Dependent Surveillance-Broadcast (ADS-B) data for surveillance, though still in its infancy, is being proven in the Bethel region of Alaska where ADS-B targets are being used by ATC in conjunction with radar returns to provide separation services ${ }^{2}$. The availability of ADS-B data also under-lies development of self- 
separation tools such as Cockpit Display of Traffic Information (CDTI) and airborne Conflict Detection and Resolution (CD\&R) functions that are fundamental to most distributed traffic management proposals. Methods of augmenting GPS such as Wide Area Augmentation System (WAAS) promise to provide approach capability at airports currently lacking ground-based approach facilities. Finally, development of aircraft technologies such as small, fuel-efficient turbofan engines, advances in aerodynamics, anti-icing methods, and avionics has resulted in a new generation of small aircraft with seat-mile costs approaching that of transport-category aircraft.

Unfortunately, many of the airports reaping benefits from these emerging technologies lie outside of existing ATC radar coverage. Providing conventional air traffic separation services at these airports would require one of the following: 1) a dramatic expansion of the ATC surveillance radar network and/or ADS-B data-link systems along with a concomitant increase in ATC personnel, or 2) reliance on workload-intensive non-radar approach procedures. The first approach is likely to be very costly, in terms of required investments in both infrastructure and personnel, but it would permit relatively highdensity operations over a wide area. The second option would require less investment in infrastructure, but it would still require additional ATC personnel, and it would be restricted to relatively low-density operations because of the limitations inherent in non-radar type operations. Since neither of these options seems particularly desirable, automated means for providing IFR separation without direct ATC intervention are being explored.

\subsection{Conventional Non-Radar IFR Procedures}

Because newly introduced procedures must function within the existing National Airspace System (NAS), their architecture must complement, and to the largest extent possible, make use of existing infrastructure and procedures. Before addressing proposals for automation of non-radar approach and departure separation services, we will review these existing procedures.

Separation services can be classified as either radar (target-to-target) or procedural (target-toairspace). The latter is used when accurate surveillance data is not available, (generally nonradar environments), or the intent of a target is unknown (VFR targets or IFR operations in uncontrolled airspace). There are also hybrid techniques, utilizing both local target separation and more general airspace structure to keep nonparticipatory aircraft separated from IFR operations. One example is a block of high altitude airspace, known as a "wave window", that allows gliders not equipped with transponders to operate in Class A Positive Control Airspace. All IFR traffic is separated from the block of airspace, and within the window, the gliders use a combination of see and avoid and specialized rules of the road to maintain their own separation from each other. ATC is responsible for the former, the pilots the latter. Similarly, the structure of ICAO-defined airspace types serves a similar purpose: to minimize the mixing of different types of traffic where possible, and ensuring the compatibility of mixed traffic by mandating equipment appropriate to each type of airspace.

In a non-radar airport environment, separation services are often provided to IFR flights by ensuring that airspace around the airport has no other IFR flights within it, i.e. the airspace is "sterile". Additional requests for operations at the airport are postponed until the IFR arrival or departure is complete, hence the name "onein/one-out". For departures, pilots are restricted to a specified departure window known as a clearance void time, during which the airspace from the departure airport to the point at which radar contact is expected or position reporting will commence is guaranteed by ATC to be sterile. If the departure window is missed, a new clearance request must be made to gain entry to controlled airspace. For arrivals, the same principles apply, though the "one-in/one-out" window is typically defined by the loss of radar contact (common in descent) and the pilot's action of closing their flight plan. If, for example, an arriving aircraft requests an approach while another aircraft was landing, the arriving aircraft will likely be required to hold at a location at a safe distance and altitude from the active approach path. They will be given an Expect Further Clearance (EFC) time when they can expect to receive clearance to initiate their approach. This procedure permits ATC to separate multiple flights in the absence of surveillance data (radar) without requiring excessive position reporting.

Many people, including licensed pilots, are surprised to learn that many airports in the US with no radar coverage or control tower have instrument approach procedures with final approach segments in uncontrolled (class G) airspace, where the pilot, not the controller, is responsible for traffic avoidance. Since the floor of 
controlled airspace near instrument airports in the US is typically only $700^{\prime}$ AGL, ATC can provide adequate separation between IFR arrivals and departures, but the possibility exists that VFR traffic of which ATC has no knowledge may be present in the airport vicinity below 700'. Regulations permit VFR flight within class G airspace in the vicinity of an airport as long as the aircraft can remain clear of clouds and maintain flight visibility of 1 mile. Because there is no practical way for an arriving IFR aircraft to seeand-avoid VFR traffic operating near the base of a $700^{\prime}$ ceiling, the only means for ensuring separation between IFR and VFR traffic in these circumstances is mutual use of the Common Traffic Advisory Frequency for position reporting. If the VFR aircraft is not radio-equipped, separation is simply left to chance. Fortunately, the low volume of VFR operations at airports in extremely marginal meteorological conditions has made traffic conflicts of this type rare.

\subsection{Automated Instrument Procedures}

There have been a number of studies aimed at developing efficient instrument access to nonradar facilities through the automation of approach and departure traffic separation procedures. Most of these attempts depend on 4-D (lateral, vertical and time determinate) flight path prediction. Tobias and Scoggins ${ }^{3}$ attempted to automate traditional IFR services by building off Tobias' earlier work, ${ }^{4}$ describing prediction of conflict-free approach paths. Using conventional separation standards, they generated airport-relative altitude, azimuth and range data from the beacon transponder systems (Mode $\mathrm{A} / \mathrm{C}$ ). From these data, the ground-based automation assigned routes of flight designed to provide sequence and maintain separation. A synthesized voice system transmitted these clearances to the participatory aircraft via VHF radio. Morgenstern and Telsch ${ }^{5}$ described a similar system intended for VFR advisories.

The most significant obstacle to deploying such a full-scale automated approach and departure system is the challenge of legally certifying it. The air traffic control system is an inherently conservative institution: even incremental changes to ATC procedures require lengthy regulatory processes. Moreover, the system has evolved though many hundreds of thousands of hours of service in all kinds of conditions, and represents the distillation of often bitter experience with a myriad operational difficulties and mishaps. The safety record of the modern ATC system is exemplary, and the procedures upon which this record is based should not be altered lightly. With this in mind, we propose a much less ambitious approach/departure automation system than has heretofore been advanced: one that is based on the existing, well-proven non-radar "one-in/oneout" IFR procedures described in Section 1.1. While this method is neither the most efficient use of the airspace, nor the most convenient for the pilot, it does have the virtues of safety, simplicity and relatively low cost. While our proposed system is limited to relatively low-density operations, it represents architecture from which more efficient, albeit more complex, full-service automated systems can be developed after operational experience has been accumulated and as demand for point-to-point operations increases.

This paper will focus on describing the elements of a basic automated approach/departure system, establishment of operational procedures, identification of the equipment and systems necessary for implementation. Consideration of the necessary navigation signal accuracy, integrity and availability for area-navigation-based (RNAV) instrument approaches are not addressed, as there is a large body of work on these subjects.

\section{Concept of Operations}

\subsection{The proposed model}

The proposed model for automating nonradar, non-tower arrivals and departures, hereafter referred to as the Automated Airport Control Volume (AACV) model, builds on the existing procedural or target-to-airspace separation archetype described in Section 1.1 and extends its use towards truly distributed air traffic control. During periods of IMC, a block of airspace (an Airport Control Volume, ACV) will be established around the airport and a local automation system will manage access so that only one aircraft will be present in the ACV airspace at a time. The automated system will further limit access to appropriately equipped aircraft that follow specified procedures. Like the existing procedural separation methods on which it is based, the AACV model provides a simple, relatively low-cost and extremely effective way to minimize the opportunity for traffic conflicts in the critical approach and departure phases of flight. Moreover, defining the ACV as extending to the surface can eliminate the small but finite possibility of a conflict between IFR and VFR traffic in 
uncontrolled airspace. Finally, the operational concept allows for growth by later allowing the participating aircraft within this airspace to provide their own separation services using a combination of procedures and specialized tools, including localized surveillance data.

Our fundamental approach to design of the $\mathrm{ACV}$ architecture and associated procedures is to place a minimum number of constraints on participating aircraft necessary for safety. At highvolume terminals, optimizing capacity is a paramount concern and rigid constraints must be imposed on traffic because the actions of a single aircraft can affect dozens of other flights, leading to disruptions and delays. At the low-volume airports suitable for an AACV, not only is optimizing capacity less of a concern, but aircraft can maneuver with relative freedom without interfering with each other. This environment is more akin to airport operations under VFR, where pilots have many degrees of freedom, yet a relatively simple set of priority rules suffices to keep order. We have therefore pursued a hybrid system of rule-based maneuvering, airborne selfseparation and ground control in order to ensure a safe and reasonably efficient system.

\subsection{System components}

The AACV system is comprised of four distinct components we will address separately. They include 1) the Airport Control Volume airspace, 2) protocols governing access to the $A C V, 3$ ) the automation system and communications necessary for managing the traffic flow in and out of the ACV, and 4) traffic management procedures in the vicinity of the ACV that provide the transition between AACV operations and the enroute structure.

\subsubsection{The Airport Control Volume}

The Airport Control Volume is similar in concept to a class $E$ surface area, though the ACV would also include the Initial Approach Fixes (IAF's) associated with approaches at the airport and would be restricted to typical initial approach altitudes (see Figure 1). In order to accommodate the FAA's basic RNAV approach, the ACV is defined nominally as 12 NM from the airport, from the surface to 2500' AGL. As with class E airspace, there is no reason for the $A C V$ to be effective when the airport weather is above VFR minima, although some of the associated equipment may prove useful as an aid to visual separation during operations in VMC when the exclusionary rules do not apply.

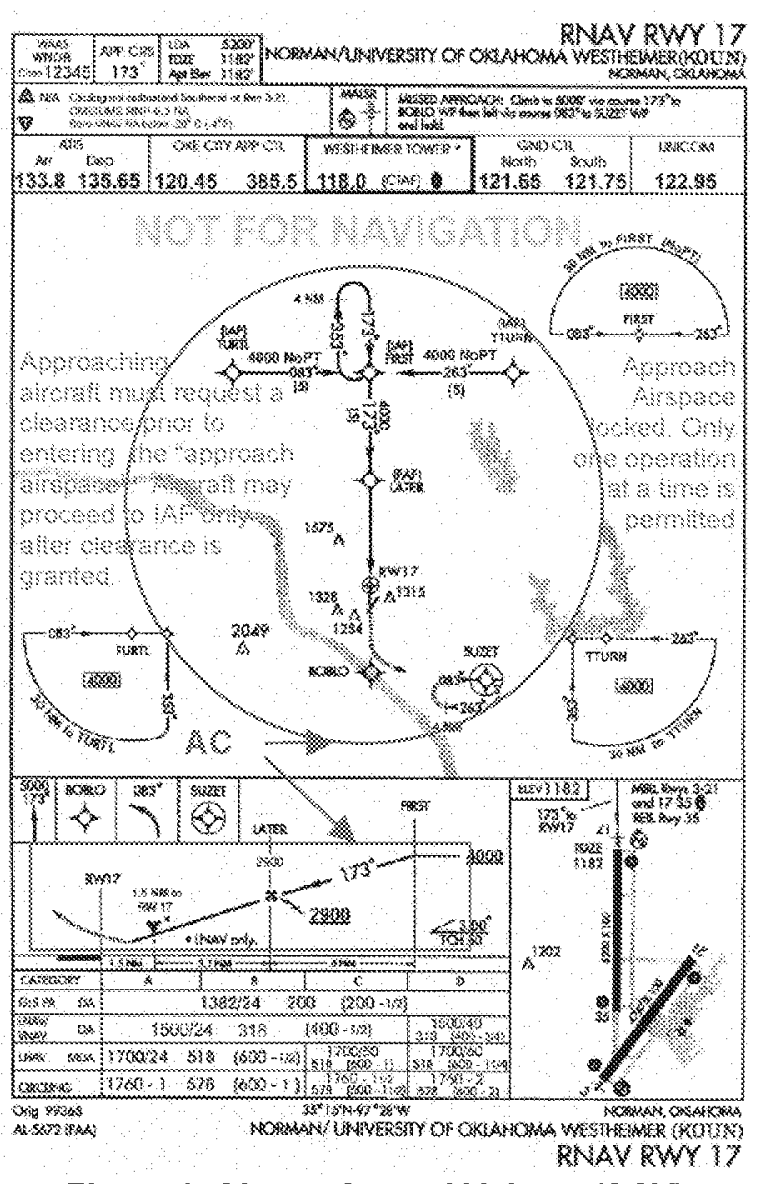

Figure 1: Airport Control Volume (ACV)

\subsubsection{Access protocols}

Arriving aircraft must request a clearance to enter the $A C V$ and they must remain clear of the ACV until clearance to enter has been granted. Either the pilot or the automation will initiate the clearance request based on a specific triggering event such as distance or time to destination. Similarly, departing aircraft must also request a departure clearance, and shall remain on the ground, clear of active runways until a departure clearance has been granted. Aircraft can also request to transition through the $A C V$ enroute to another destination.

Only one aircraft (referred to as the "priority aircraft") at a time will be granted access to the ACV by the automated system. Aircraft will be granted access to the ACV by the automated system in order of their priority as established in accordance with a mutual exclusion protocol that locks all aircraft out of the approach/departure airspace while it is in use by the priority aircraft. Once the priority aircraft is clear of the $A C V$, sequence is reestablished among all known 
requests, and the new priority aircraft will receive an automated clearance message granting access to the ACV.

Although the specifics are a matter for further study, the protocol design will need to accommodate special circumstances arising from events such as aborted takeoffs and missed approaches. There are a number of ways these events can be accommodated, but for the present study it suffices to recognize that the ACV will have to remain locked until the situation is resolved.

The priority list is generated by a sequencing protocol in the local automation system based on requests for approach, departure or transition operations. The ranking algorithm will take into account a number of factors in creating the ranking. Even with the simple "one-in/one-out" operational model, it is possible to order requests to make better use of the airport resource. A simple study of arrival sequencing scenarios showed as much as a $46 \%$ decrease in the summative delay taken by all participating aircraft by assigning priorities to all requests received within a particular period on the basis of airport usage rather than in the order the requests were received. The type of operation, the position of arriving aircraft in relation to the IAF's, aircraft ground speed and the available approach profiles must all be considered in determining the ranking which make most efficient use of the airspace because each has an effect on the duration of the requested operation. For example, it may be more efficient to assign a departure or a transition request (which are relatively quick operations) a higher priority than an approach request that was received earlier.

In practice, reordering the priority list in the interest of efficiency should, however, be limited to inserting small numbers of departure and transition operations between previously ranked arrival requests and deferring departure requests when predicted holding time for arrivals becomes excessive; the ranking of arrival requests should not be altered in relation to each other. The reason for this is simple: arriving pilots need some idea when they can expect to receive an approach clearance in order to make potentially critical decisions regarding fuel management and diversion to an alternate destination. After pilots have been advised of the EFC time, the relative order of arrivals must be preserved in order for this information to remain valid.

Although the priority list is created on the basis of valid clearance requests by aircraft in the immediate vicinity of the $A C V$, it may also be advantageous to develop forecasts for airport demand that can be used to generate delay estimates well in advance. Flight plan data, required enroute updates and data from the FAA's HOST computer or other ground-based surveillance systems can be used by the AACV automation to periodically compute and update predictions of the sequence of arriving aircraft, periods when the airspace may not be available for departures, or when overall demand on the airport exceeds its capacity. This information can then be disseminated in the form of pilot advisories.

\subsubsection{Automation system and supporting message exchange}

As noted above, all automated communication, sequence generation and clearance granting functions are performed by a local AACV data processing system. All participatory aircraft will be required to be ADS-B equipped, with a 40-mile transmission capability (although preliminary studies suggest that a transmission capability as low as $\mathbf{2 0}$ miles may be sufficient). ADS-B will provide the primary means of communication between the aircraft and the AACV system, providing general data transfer and surveillance functions. For our initial implementation, we have attempted to use a minimal message set, assuming substantial cost for all transmitted data.

For Approaching Aircraft: A specific oncondition request report would be designated, containing Aircraft ID, intended airport facility, and request code (landing or transition). This message could be generated by the on-board flight planning and/or navigation avionics at a specified point on the flight path (e.g. $20 \mathrm{NM}$ to destination). "Priority granted" messages will be transmitted to the aircraft through data link and will consist of airport ID, aircraft ID, time, IAF assignment, and a single priority bit. "Priority denied" messages will consist of airport ID, aircraft ID, EFC time, and a single denial-of-priority bit. The denied aircraft would re-request at the EFC time to minimize message traffic. This time is also useful in the event of lost communications. An "operations complete" message could be used to unlock the airspace. Optionally, ADS-B surveillance data transmitted from the priority aircraft indicating the aircraft's velocity has dropped below a specified threshold for a specified duration could perform this function. State data as described by RTCA DO $-242^{6}$ (Time, 
Lat, Long, altitude, ground-referenced velocity vector, ID, category, navigational data quality) will be sufficient input for the ground-based arbitrator to calculate priority.

For Departing Aircraft: As for arrivals, departures would use the specific on-condition request report, containing Aircraft ID, intended airport facility, and request code (departure). "Priority granted" messages will be received through data link, and will consist of airport ID, aircraft ID, time, departure runway, and a single priority bit. "Priority denied" messages will consist of airport ID, aircraft ID, EFC time (or equivalent, as discussed in Section 2.2.2), and a single denial-of-priority bit. The denied aircraft would rerequest at the expect further clearance time to minimize message traffic. An "operations complete" message could be used to unlock the airspace or ADS-B surveillance data transmitted from the priority aircraft indicating aircraft position outside the $A C V$, could perform this function. State data will be sufficient input for the ground-based arbitrator to calculate priority

\subsubsection{Traffic separation outside the ACV}

Another significant operational element necessary for a feasible system is some form of traffic separation in the vicinity of the ACV. While the AACV concept is primarily intended to compliment the development of self-separation capabilities, it could be implemented within the existing ATC architecture. In such a case, ATC would likely provide separation outside of $A C V$ by controlling arrivals and assigning each aircraft a discrete holding area where it would be required to remain until clearance into the $A C V$ is granted.

When self-separation tools such as CDTI and CD\&R become available, the simplest approach would be to require pilots to use these tools to provide their own separation from other aircraft operating in the vicinity of the ACV. A number of studies have suggested that pilots can be provided appropriate tools to enable selfseparation in these circumstances $7,8,9$, but the matter requires careful consideration. While the effectiveness of self-separation tools in the enroute environment has been demonstrated, the application of these tools to a situation where multiple aircraft are attempting to execute holding maneuvers in close proximity is less well established. Aircraft trying to remain close to a particular point are constrained in a way that aircraft enroute are not. Moreover, the pilot workload immediately in advance of executing an approach is significantly higher than during the enroute phase, leaving less time to attend to traffic avoidance.

It is beyond the scope of this study to establish specific requirements for separation assurance outside the $\mathrm{ACV}$, but further research must be undertaken to answer this question before a complete description of an operational AACV system is possible. It may turn out that additional constraints must be placed on selfseparating aircraft outside the $A C V$ akin to the holding assignments used by ATC today, but to do so would be to remove some of the simplicity inherent in the AACV concept and advance the system significantly closer to a full-scale automated traffic control system.

\subsection{Non-normal Operations}

Existing procedures have evolved to accommodate a wide range of non-normal operational situations. Fortunately, most of these procedures translate readily into the AACV concept. The most significant abnormal situations are discussed below.

\subsubsection{Lost Communications: Aircraft Avionics Failure}

As in other "lost com" situations in today's system, a pilot who is unable to report an updated estimated time of arrival (ETA) is required to try to honor their last-reported ETA. If they are running early, they reduce speed to adjust their arrival time. If they are running late however, they may miss their window and have no way to make up sufficient time. The AACV system would have no way to know if they were truly just late or had gone "lost com" and are actually in the vicinity of the AACV, but not reporting. Whenever failure of an aircraft to make a mandatory report or the loss of ADS-B data suggests that an aircraft has experienced avionics failure, the AACV system would respond by locking the $A C V$. Of course, if ADS-B surveillance data for the lost-com aircraft later becomes available to the AACV system indicating the aircraft has not yet arrived in the airport vicinity, some operations could be resumed, if it is clear they can be completed before the lost-com aircraft arrives.

Federal regulations preclude extended IFR operations without ATC communication capability. $^{10}$ If VMC exists, pilots are expected to land "as soon as practicable". In the case of avionics failure enroute to or around an AACV airport in IMC, the following guidelines derived from the existing regulations could pertain: If access to the ACV has been granted prior to avionics failure, the flight would immediately 
proceed to the appropriate IAF to initiate the approach and landing. If access to the ACV was denied prior to avionics failure, the flight would remain outside the $A C V$ until the EFC time received with the denial message, and then proceed to the appropriate IAF to initiate the approach and landing. In either case, the ACV would remain locked until the aircraft is confirmed by ATC to have landed, diverted to another airport or departed the area. The AACV would then be manually reset for normal operations.

\subsubsection{Lost Communications: AACV Failure}

From an aircraft's perspective, if an approach clearance request is made but no response is received, there may be no immediate way to tell if there is a communication failure in the aircraft transceiver or a failure of the ground equipment. Before following procedures for lost communications, it would be prudent for the pilot to try to determine if it is the airport reception or their transmission/reception that has failed. A test message could be included in the message set for this purpose, or facilities at another nearby airport may be of use. If the pilot can confirm that the airport facility has lost capability, an approach under IFR using these procedures should not be initiated. If an approach clearance has already been granted when airport automated sequence communication is lost, a pilot should be able to complete their approach assuming they still have appropriate approach guidance signal.

\subsubsection{Urgency and Emergency Situations}

Extended arrival delays resulting from high demand can cause problems for some operators. Weather and mechanical problems can cause emergencies to arise at any time. The AACV can automatically accommodate many of these situations by the use of a priority word in the oncondition ADS-B message. We could use 3 bits to describe four levels of urgency, granting clearance according to priority first, then position/time precedence:

0) Normal Operations. Sequence generated as described in Section 2.2.2.

1) Re-request. Set only by system, not user selectable; priority supercedes initial transition and departure requests.

2) Pilot-initiated Urgency request. e.g. Low fuel state; requesting aircraft assigned next priority status; "priority granted on basis of urgency request" message transmitted to ATC.

3) Emergency. e.g. Declaration of an in-flight emergency; priority status of current "priority aircraft" rescinded if practical (for instance, priority aircraft has not yet entered the $\mathrm{ACV}$ ) and earliest possible clearance of emergency aircraft for the approach; "declaration of emergency" message transmitted to ATC for investigation.

\subsection{Extensions Beyond the One-In/One-Out Concept}

The next step in the development of automated approach and departure procedures, allowing more than one aircraft into the ACV at a time, depends on tools which enable pilots not only to self-separate, but also to order and merge themselves on or near the approach. SelfSpacing concepts under development at NASA Langley may provide such aids ${ }^{11}$. These tools may help pilots adjust their flight using speed and path guidance generated from interval and targetreferenced data rather than ground-referenced instrumentation. This would allow a pilot to effectively fly a similar guidance cue to today's course deviation indicator, but by maintaining both course and speed guidance, they would also be assuring separation from the reference target as confirmed by the cue-generating automation.

\subsection{Regulatory considerations}

As this procedure's main purpose is to provide separation assurance, all of the airspace within an ACV would necessarily be defined as controlled airspace in order to exclude non-participatory traffic. Mixing AACV operations with standard IFR operations cannot occur without full surveillance of all traffic targets, or a means to share IFR airport usage/cancellations data between ATC and the AACV. Preferably, the AACV will have surveillance data for all traffic targets in the area. Assuming an ADS-B-based system, mandatory use of ADS-B transmitters in the vicinity of an active $A C V$ would be one solution. Alternatively, a combination of ground-based passive (or active Mode- $C$ based) technology and airborne reception equipment could supplement those targets not ADS-B equipped. In fact, these types of systems could be the primary means of surveillance, though they would most likely be cost prohibitive for many municipal facilities.

A new subset of clearance descriptions within Instrument Flight Rules would be necessary as well. Regulations regarding mandatory equipment for participating aircraft already provide for this type of growth, as "two way radio communications and navigational equipment appropriate to the ground facilities" are obligatory ${ }^{12}$. Clearly, these types of approaches may require a whole host of new airborne equipment, including self-separation tools (appropriate surveillance, CDTI, CD\&R, etc) 
and a method to exchange data and clearance information with the sequence granting authority at the airport facility.

\section{Preliminary simulation}

The AACV model combines a variety of new and existing technologies and procedures, many of which have been studied independently of the others. There are a number of people studying the ability of flight crews to self-separate using tools such as CDTI and CD\&R. The use of ADS-B for surveillance, though still in its infancy, is also not novel: in addition to the Capstone project mentioned earlier, the FAA's Safeflight 21 program $^{13}$ and the North European ADS-B Network or NEAN/NUP project ${ }^{14}$ have collected a great deal of in-flight ADS-B performance data. There is also a lot of work on the use of GPS and GPS augmentation schemes such as WAAS to provide approach capability at airports currently lacking an instrument approach. The concepts of procedural separation and the one-in/one-out use of a volume of airspace near an airport are well proven in today's NAS. Since we were able to draw on this large body of research and operational experience, we have developed a batch simulation to validate the systemic attributes of the AACV operational concept.

The sequencer components of this batch simulation are designed to be compatible with pilot-in-the-loop simulations developed at NASA Langley $^{15}$ for study of pilot workload and the evaluation of new procedures. These same software elements can be used in conjunction with other simulation elements for pilot/controller studies to validate procedures developed for transition from traditional approaches and operations to these automated procedures. A combination of this batch processing capability and piloted simulations are needed to identify improvements in the operational concept necessary for further development of the AACV.

\subsection{Simulation Description}

The automated airport simulation has four primary functions: traffic generation to introduce aircraft into the environment with an appropriate mix of initial conditions, trajectory estimation (and therefore calculation of time on approach), a sequencer for the determination and dissemination of sequence, a delay function that will insert service re-requests from aircraft initially denied service, and data collection.
The automated airport operation system was modeled as a single server queue as shown in Figure 2.

Aircraft are randomly introduced to the simulation based on an exponentially distributed average inter-arrival time, $\lambda$. Aircraft position at request time is assigned randomly within an annulus designated by the outer limits of the ACV plus some maneuvering space and the modeled ADS-B reception limit. An appropriate preferred IAF is assigned by the simulation based on geometric position relative to a standard $T$ RNAV approach as defined by the FAA. ${ }^{16}$

Approach arrivals are assumed to fly direct to the IAF and then initiate the approach. For our purpose of exploring a mix of approach speeds, three aircraft types are used; a light single engine piston, an Eclipse Jet, and a Transport/Regional jet. Arrivals are assigned a speed profile based on type. The operation duration is then calculated for each aircraft in the simulation as $f$ (path, speed).

Sequencer events consist of aircraft approach and departure clearance requests, though only unscheduled approaches have been implemented to date. An arrival can find the system locked (airport in use) or unlocked. If the system is free, requests are en-queued for a short time (a study variable) and one aircraft is selected for service (given priority). The rest of the requests in the queue are rescheduled and merged with the arrival stream with a higher priority, an updated position and a re-request time. The re-request positions can be modeled differently than initial requests because delayed aircraft may be more likely to re-request while near an IAF (altitude separated from the initial approach altitude) rather than scattered randomly about the airport perimeter. The simulation can model the rerequest arrivals either way. Figure 3 shows a typical 10 hour-aggregate traffic sample as generated.

If necessary, charted holding could be added to the procedure to facilitate safe and easily selectable waiting areas for delayed aircraft. Holding has not been modeled, as our purpose was to determine the size and scope of delays we could expect given differing rates and mixes of arrival traffic. Human-in-the-loop studies will be necessary to determine feasibility of free flight and self separation in this region vs. a more 


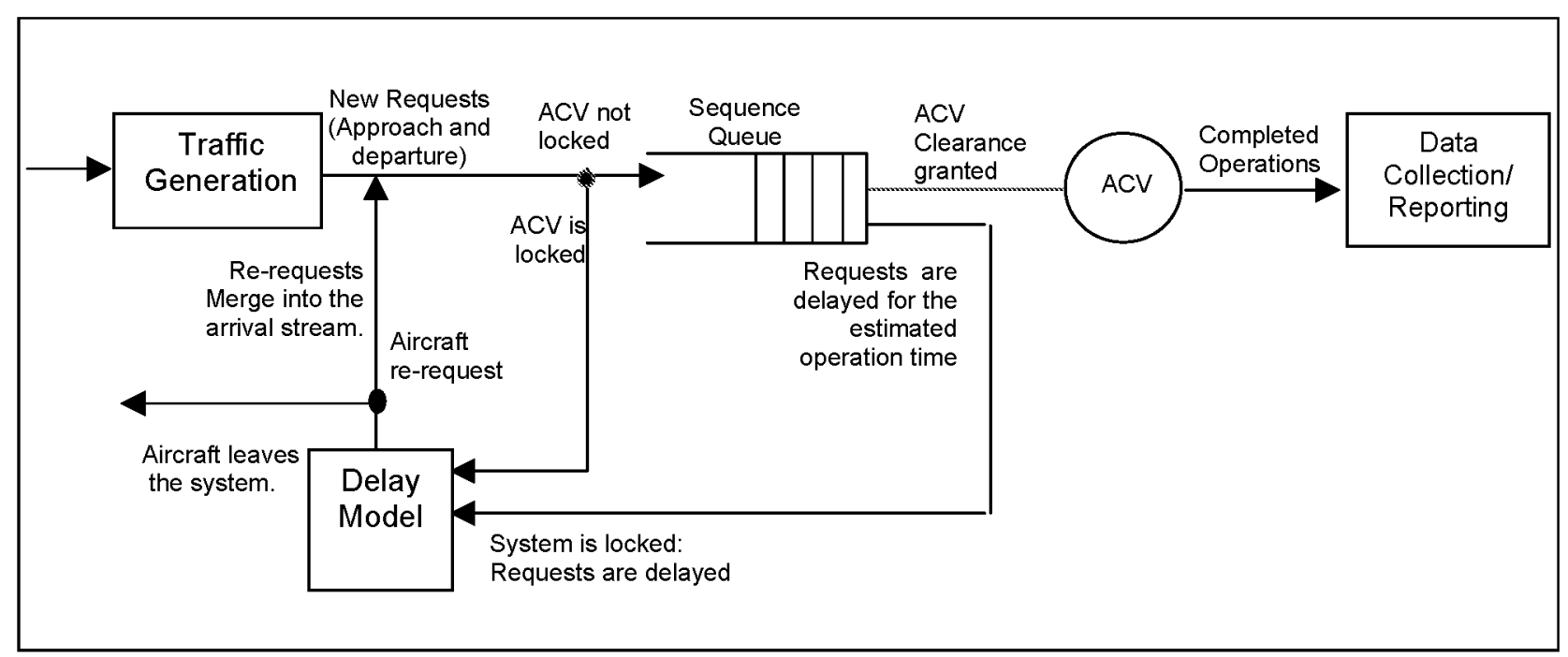

Figure 2: Sequencer Model in Simulation

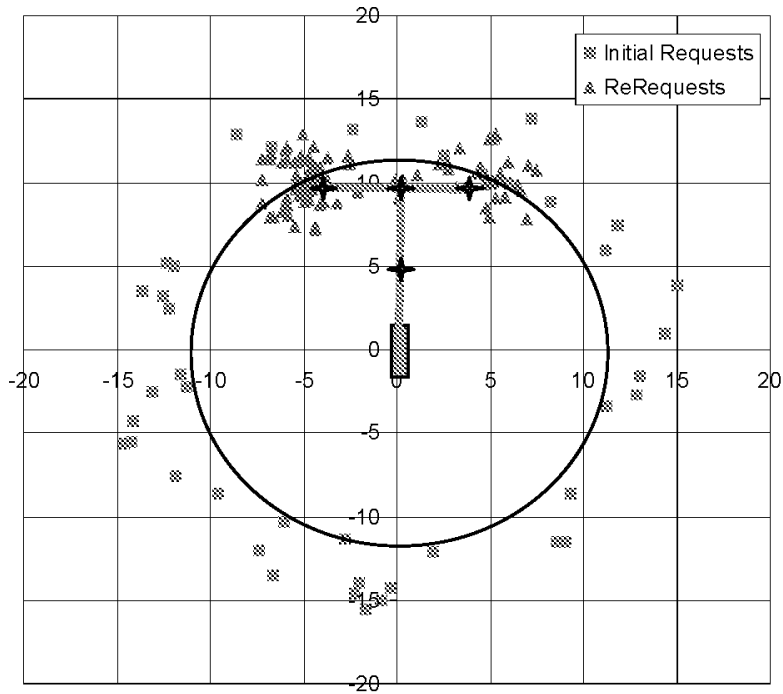

Figure 3: Typical Arrivals (10 hr Aggregate)

constrained, planned hold or automated path stretch vector assistance as others have suggested in the references above.

Provision of more information to the returning aircraft than simply when the airport was expected to reopen was found to decrease queue lengths (and is useful in non-normal operational considerations). By providing an estimate of a specific aircraft's usage time (implying implicitly or perhaps explicitly sequence position as well), message traffic in the form of requests and responses was decreased dramatically, on the order of $75 \%$.

\subsection{Experiment Design}

A six factorial response surface text matrix was generated, varying the size of the sequence queuing window, the lateral limits of the ACV boundary, the outer limits of ADS_B reception (and therefore when an approach request could be received), the average arrival Rate $(\lambda)$, the time of re-request relative to the estimated airport use time, and the mix of aircraft (approach speeds). Independent variable levels chosen to be representative of the design space can be seen in Figure 4.

\begin{tabular}{|l|l|r|r|r|r|r|}
\hline $\begin{array}{l}\text { Independent } \\
\text { Variable }\end{array}$ & Units & \multicolumn{5}{|c|}{ Levels } \\
\hline Request window & $\min$ & 0.1 & 0.7 & 1.1 & 1.4 & 2.0 \\
\hline ACV boundary & $\mathrm{NM}$ & 12.0 & 14.6 & 16.0 & 17.4 & 20.0 \\
\hline ACV annulus & $\mathrm{NM}$ & 3.0 & 8.5 & 11.5 & 14.5 & 20.0 \\
\hline Arrival interval $(\lambda)$ & $\mathrm{min}$ & 4.0 & 22.1 & 32.0 & 41.9 & 60.0 \\
\hline Re-request delay & $\mathrm{min}$ & 0.0 & 0.7 & 1.0 & 1.4 & 2.0 \\
\hline$\% \mathrm{GA}$ & $\%$ & 1 & 33 & 51 & 68 & 100 \\
\hline
\end{tabular}

Figure 4: Variables Limits

Using a balanced central composite experimental design, 86 samples, each simulating 10 hours of airport arrivals were taken. For each sample, the simulation was allowed to continue to run until all arriving traffic had been serviced. Arrival data were collected for selected responses and averaged over the sample period. A summary of the data is shown in Figure 5.

\subsection{Results and Discussion}

Some delays are inevitable due to the nature of the service an automated one-in-one out airport would provide. Before we can field such a system, we must determine what level of delay is operationally feasible. By looking at the summary statistics 


\begin{tabular}{|l|r|r|r|r|}
\hline Response & Mean & Median & \multicolumn{1}{c|}{ Min } & \multicolumn{1}{c|}{ Max } \\
\hline Sim Duration & 616.4 & 600.0 & 600.0 & 1446.9 \\
\hline Airport Utilization & $44 \%$ & $43 \%$ & $15 \%$ & $84 \%$ \\
\hline New Requests & 21.7 & 20.0 & 6 & 129 \\
\hline Total Repeat Arrivals & 28.8 & 11.5 & 1 & 1187 \\
\hline Total Sequence Queue & 30.0 & 22.0 & 6 & 525 \\
\hline Max Sequence Queue & 2.7 & 2.0 & 1 & 36 \\
\hline Total in Holding & 11.4 & 8.5 & 1 & 120 \\
\hline Max In Holding & 3.1 & 2.0 & 1 & 72 \\
\hline Highest Priority & 4.0 & 3.0 & 2 & 32 \\
\hline Avg Wait & 11.5 & 6.3 & 1.5 & 361.3 \\
\hline Max Wait & 49.2 & 26.8 & 5.5 & 1341.6 \\
\hline Ave Operation Duration & 13.2 & 13.0 & 9.4 & 18.9 \\
\hline
\end{tabular}

Figure 5: Summary Data

alone, one can see that the simulation predicted large delays under certain circumstances, but that minimal delays and few re-requests were likely for more typical, small-airport characteristics.

An ANOVA analysis of the data showed significant correlation between a few independent variables (e.g. \% GA) and important measured responses (e.g. airport utilization and average wait time), but the effects of $\lambda$, the inter-arrival time, were strongly significant and dominated the effects of the other variables. This result confirms what one might intuitively expect: as the average interarrival period nears the average operation duration, the queues in the system begin to build dramatically. The relatively mild correlation between the other variables and the performance of the system implies that wide latitude can be taken in the design of a particular AACV without adversely affecting the performance outcome. It was also apparent from the simulation results that the system is robust enough to handle occasional traffic spikes that might be expected to occur even at a low-use airport.

The model (see Figure 6) of Utilization= $f(\% \mathrm{GA}, \Lambda$, request response time) had $\mathrm{F}=82.16$, p. $<.0001$ showing significance at the 0.05 level. The request response time proved to be a small effect as compared to the environmental factors of traffic mix and rate of arrivals.

The model (see Figure 7) Average Delay= $f(\% \mathrm{GA}, \lambda)$ had $\mathrm{F}=28.53, \quad$ p. $<.0001$ showing significance at the 0.05 level.

One other observation warrants mention: the operation time for each priority aircraft's use of the ACV was estimated using a simplified path predicated on random position assignment and IAF selection. The only independent variable directly influencing the time to fly a specified path is the assigned type (and therefore speed profile) as determined by the independent variable \%GA,

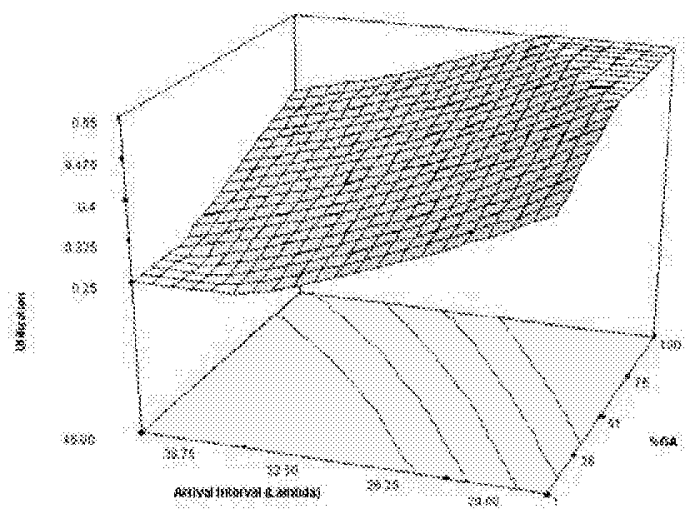

Figure 6: Utilization $f(\lambda, \% \mathrm{GA})$

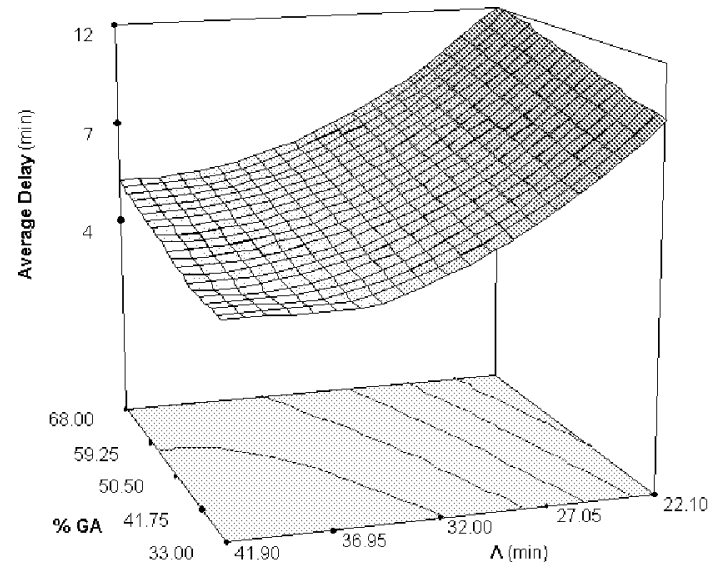

Figure 7: Average Delay $f(\lambda, \% G A)$

yet in the analysis of the response Average Operation Duration, $\lambda$ again appears significant. Though not immediately obvious, the result is due to the system's preferential selection of shorter operations, and the increasing availability of a range of predicted operation times as the queues grow with decreasing inter-arrival times (Figure 8).

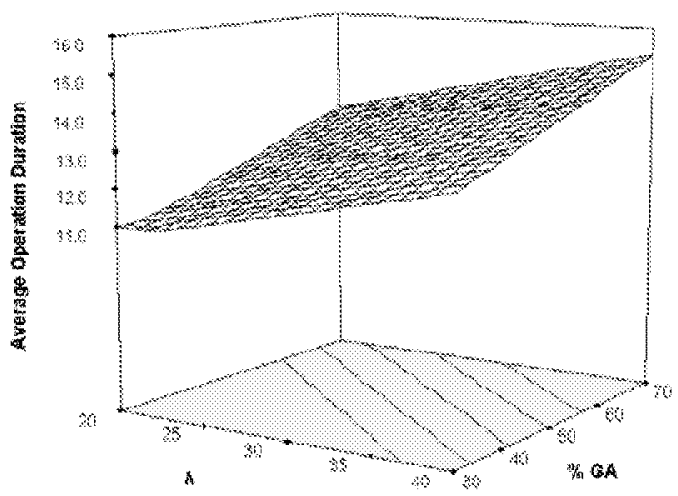

Figure 8:

Average Operation Duration $f(\lambda, \% G A)$ 


\section{Conclusion}

Some years ago a bumper sticker reading "THINK GLOBALLY, ACT LOCALLY" was popular. It was understood to mean revolutionary social change can be accomplished through action at the grass roots level, even when attempts to impose change from above are sure to fail. Removed from its political context, this notion has some relevance to the task of revising the air transport system. Structural changes such as significantly increasing the mobility of the system, or instituting technological innovations like distributed air traffic management or automating air traffic control functions represent revolutionary change; to impose these changes on a large-scale or systemwide basis would be a difficult and risky proposition at best. The alternative is to demonstrate new methods and procedures initially on a small scale, taking great care to conform to the fullest possible extent to the existing regulatory and procedural framework. If the new techniques are found to have merit on the basis of operational experience, they can be replicated elsewhere in the system and extended to largerscale applications on an incremental basis.

The AACV concept is an ideal model for implementing a number of highly innovative technologies and procedures in a low-cost, safe and conservative manner. An operational AACV would be compatible with today's ATC structure, but it would readily accommodate the introduction of self-separation technologies and it represents a platform upon which more sophisticated automated approach and departure traffic management systems could be deployed. Experience with these systems in the low volume environment of the AACV could be used to validate the technology before attempts are made to adapt these systems to higher-volume terminal areas.

Analysis of the results of a purpose-built simulation has validated the basic premise of an $A A C V$ operating under one-in-one-out protocols at low traffic volumes. The next step in the development of the AACV concept involves additional simulation studies to resolve a number of issues such as traffic management outside the AACV. This research would be followed by development of a full-scale system simulation of sufficient fidelity to permit pilot-in-the-loop studies. If the concept continues to show promise, a field study involving an operating AACV system prototype should be undertaken at a suitable airport. All of this research can be conducted relatively quickly and at reasonable cost because of the small-scale nature of the proposed system.

1 Mineta, N. Secretary DOT Testimony before Commerce, Science and Transportation Committee of the U.S. Senate, Jan. 2001

2 FAA AUA-600 Capstone ADS-B Evaluation Acceptability Memo, US DOT, Dec. 2000

3 Tobias, L. and Scoggins, J.L. Time-Based Air Traffic Management Using Expert Systems. NASA TM-88234, Ames Research Center, April 1986

${ }^{4}$ Tobias, Automated Aircraft Scheduling Methods in the Near Terminal Area, AIAA 72-120, Jan. 1972.

${ }^{5}$ Morgenstern, B. and Telsch, R. A Description of the Phase I Automated Terminal Services Concept, FAA-EM-76-6, Nov. 1976.

${ }^{6}$ RTCA Special Committee 186, Minimum Aviation System Performance Standards for Automatic Dependent Surveillance Broadcast (ADS-B), RCTA DO-242, Feb. 1998.

7 Ebi M., A Self-Organizing Approach for Resolving Air Traffic Conflicts, The Lincoln Laboratory Journal Vol 7, No 2, 1994.

${ }^{8}$ Hoekstra, Ruigrok, and vanGhent, Free Flight in a Crowded Airspace?, $3^{\text {rd }}$ USA/Europe Air Traffic Management R\&D Seminar, June 2000.

${ }^{9}$ Wing, D.J.et al, Airborne Use of Traffic Intent Information in a Distributed Air-Ground Traffic Management Concept: Experimental Design and Preliminary Results, NASA/TM-2001-211254

${ }^{10}$ FAR 91.185

11 Williams, D.H. Time-Based Self-Spacing Techniques Using CDTI During Approach To Landing in a Terminal Area Vectoring Environment, NASA-TM 84601, April 2000

${ }^{12}$ FAR 91.205 (d) (2)

${ }^{13}$ Cargo Airline Association and FAA Safeflight 21 Office. Phase 1 Operational Evaluation Final Report, Wilmington, Ohio. April 2000

14 North European ADS-B Network Update Program, NUP. htro:/hmm nuo nu

${ }_{15}$ Peters, M. and Ballin, M. A Multi- Operator Simulation for Investigation of Distributed Air Traffic Management Concepts AIAA-2002-4596, Aug. 2002

${ }^{16}$ Federal Aviation Administration, Terminal Arrival Area Design Criteria, FAA 8260.45A, July, 2000 